\title{
Research on Distribution Characteristics of Primary and Secondary School Sports Grounds in Nanchang City Based on GIS Technology
}

\author{
Hui Xiao \\ Jiangxi Science \& Technology Normal University, Jiangxi Province, Nanchang, 330013
}

Keywords: distribution characteristics, primary and secondary school, sports grounds, Nanchang City, GIS technology

\begin{abstract}
Primary and secondary school students are a country's future development hope, and their physical health is worthy of causing the government to attach great importance to it. Sports is an important means to ensure physical health, and the distribution of sports venues is of great significance to mobilize the enthusiasm of primary and secondary school students to participate in sports activities and to promote their formation of good physical activity habits. Therefore, it is particularly important to scientifically plan the distribution of primary and secondary school sports fields. Taking Nanchang City as an example, this paper studies the distribution characteristics of primary and secondary school sports grounds in Nanchang based on GIS technology, which can be used for reference.
\end{abstract}

\section{Introduction}

Adolescents are the foundation for ensuring the long-term nationality of a country. Their physical health directly reflects the development of a country's sports industry. The Third Plenary Session of the 18th Central Committee has clearly pointed out that the national public should pay attention to extracurricular exercise and physical exercise. However, according to the survey, the physical health status of primary and middle school students in Nanchang is not ideal. The reason is mainly due to the lack of physical exercise. The sports ground is an important factor affecting the interest and awareness of primary and middle school students participating in physical exercise. Therefore, it is of vital significance to explore the distribution characteristics of primary and secondary schools in Nanchang.

\section{Overview of GIS Technology}

Geographic Information System (GIS) belongs to a comprehensive discipline, which involves remote sensing science, geography, computer science and cartography. It belongs to a computer data processing system, which can save, display and query geographic data [1]. GIS is a technical system that analyzes and processes some geographically distributed data in some or even the entire surface space of the Earth by means of computer software and hardware. It is mainly used for various geospatial entities such as remote sensing image data, positioning data, attribute data and graphic data. The management and processing of data and its relationships enable the analysis and processing of various phenomena and processes distributed within a geographical area to provide a reliable basis for management and planning. At the same time, GIS technology can realize real-time monitoring of resource and environmental conditions and changes in production activities in different periods, and analyze and contrast them. With its network analysis function and spatial analysis function, and then formulate relevant evaluation indicators, various groups can be judged. Whether the accessibility to specific social service facilities is balanced, and whether the distribution of various resources is appropriate is of great significance for energy-saving resources and promoting sustainable development of society [2]. 


\section{The Distribution of Sports Grounds in Primary and Secondary Schools in Nanchang Based On GIS Technology}

\subsection{Selection of Evaluation Indicators.}

In the past, the primary and secondary school sports venues in Nanchang were mainly for primary and secondary school students. In 2017, the government introduced some policies. Some Nanchang primary and secondary school sports venues began to open to the public, that is, their service targets are no longer restricted to primary and secondary school students, but to the general public. . To this end, this paper aims at the characteristics, functions, technical operability and availability of statistical indicators of the primary and secondary school sports venues in Nanchang City. Based on the geospatial perspective, the evaluation index of the sports ground distribution is set to be consistent with the population distribution. According to the per capita investment amount, per capita site area and the number of sports venues per 10,000 people, the primary and secondary school sports fields are assessed to be consistent with the population distribution. At the same time, the distribution of sports grounds in primary and secondary schools is assessed according to the amount of site investment, site area and number of venues. Is it reasonable [3].

\subsection{Calculation Method for Evaluation Indicators.}

One of the more widely used methods in spatial analysis of GIS systems is to test whether the distribution of two geographical things in space is consistent, which can be measured by the following formula:

$$
\Delta P=\frac{\sum_{i=1}^{n}\left|\frac{P_{i}}{P}-\frac{S_{i}}{S}\right|}{2} \times 100 \%
$$

Where S represents the sum of the areas; Si represents the area of each area; P represents the sum of the population; and Pi represents the population of each area. If it is close to 1, it means that the population distribution is uneven and excessively concentrated in a certain area. At this time, it can be measured by the following formula (2); if it is close to 0 , it means that the same proportion of land area is distributed with the same proportion of people, land. The area is consistent with the population distribution, that is, the population distribution is even.

$$
R_{X Y}=\left[1-\frac{1}{2} \sum_{i=1}^{\wedge}\left|\frac{X_{i}}{\sum X_{i}}-\frac{Y_{i}}{\sum Y_{i}}\right|\right] \times 100 \%
$$

Represents the total scale of the sports field (investment amount, site area, quantity) and population distribution consistency index; Y represents the sports ground in the administrative area; represents the sports ground in the corresponding area; $\mathrm{X}$ represents the total population in the administrative area; Total population. If it tends to 0 , it means that the sports grounds are concentrated in some areas, and the distribution is not balanced. If it tends to 100, it indicates that the sports field and population distribution are in good agreement. (Note: The sports venues referred to in this article include investment amount, site area and quantity.)

\subsection{Calculation Method for the Concentration of Sports Space in the Stadium.}

In this paper, the Herfindahl index is used to calculate the distribution of sports grounds. The Herfindahl index can be calculated by the following formula:

$$
H H I=\sum_{i=1}^{N}\left(\frac{X_{i}}{X}\right)^{2}=\sum_{i=1}^{N} S_{i}^{2}
$$

Where HHI stands for Herfindahl's index; N stands for the total number of regions; S represents 
the total size of the entire regional sports ground, ie the amount of investment, the size and number of the venue, etc.; the size of the stadium representing the i-th region. If the regional sports fields are evenly distributed, the Herfindahl index is $1 / \mathrm{n}$; if all the sports fields are concentrated in a certain area, the Herfindahl index is 1, which shows that the Herfindahl index is between 1 Between $/ \mathrm{n}$ and 1 , the smaller the value, the more uniform the site distribution. According to the relevant regulations of the US Department of Justice and the Federal Trade Commission, the Herfindahl index exceeds 1800 to represent a high degree of market concentration; between 1000-1800, it means that the market concentration is moderate; below 1000, it means that the market concentration is low [4].

With the help of GIS technology, this paper uses the map of Nanchang City published in 2017 as the base map of the map, using the map data scale of 1:50000, applying the geographic information system ArcGIS9.2 software to construct the attribute layer, and investing the amount of investment in the small and medium-sized stadiums in Nanchang. The relevant data such as the site area and the number of venues were imported into this map, and corresponded to the relevant attribute layers one by one, and the spatial database of the primary and secondary schools in Nanchang City was established. After that, the relevant data is exported, and the visual patterns of the location space and the spatial distribution of the stadiums in the primary and secondary schools are drawn.

\section{Evaluation of the Distribution of Sports Grounds in Primary and Secondary Schools in Nanchang City}

The data on the number of sports venues was substituted into the formula of agglomeration. It was found through calculation that the overall HHI index of the number of primary and secondary schools in Nanchang was 659, and the concentration was low. Among them, the number of sports venues in township primary and secondary schools is 781, which belongs to the low concentration area. The number of sports venues in each township is relatively balanced. The number of sports fields in urban primary and secondary schools is 1659, which belongs to the moderate concentration area, including Donghu District and West Lake. There are a large number of primary and secondary school sports venues in the district, Honggutan New District, Xinjian District and Nanchang County.

The data on the area of the stadium is substituted into the formula of the agglomeration. It is found through calculation that the HHI index of the primary and middle school sports fields in Nanchang is 827, and the concentration is low. Among them, the HHI index of the sports area of primary and secondary schools in townships is 923, which belongs to the low concentration area. The sports grounds of primary and middle schools in Changchun Town and Liantang Town are larger, and the distribution of other towns and towns is more balanced. The HHI index of urban primary and secondary school sports fields is 1204. It belongs to the moderate concentration area, and the primary and secondary school sports areas in Donghu District, Xihu District and Honggutan New District are large.

Substituting the relevant data of sports field investment into the formula of agglomeration, it is found through calculation that the HHI index of the sports field investment in primary and secondary schools in Nanchang is 1876, and the concentration is high. Among them, the HHI index of sports field investment in township primary and secondary schools is 2743 , which belongs to the high concentration area. The number of sports fields in each township is relatively balanced. The HHI index of urban primary and secondary school sports investment is 5267, which belongs to the high concentration area, Donghu District and Xihu District. There are more sports investment in primary and secondary schools in Honggutan New District and New District.

According to the calculation of the consistency of sports field and population distribution, it is found that the sports investment amount, site area, site number and population distribution consistency index of primary and secondary schools in Nanchang are 47.69, $66 . .97$ and 83.44 respectively. It can be seen that the investment and population distribution of primary and secondary schools in Nanchang City is extremely uneven, the site area and population distribution are moderately consistent, and the number of sites and population distribution are consistent. 


\section{Conclusion}

In summary, the concentration of sports investment in primary and secondary schools in Nanchang is relatively high, and the concentration of townships is lower than that in urban areas; the concentration of site area is lower, the concentration of townships is lower than that of urban areas; the concentration of sites is low, and the concentration of townships is low. In addition, the consistency of investment, area, quantity and population distribution of primary and secondary schools in Nanchang City is uneven, moderate and relatively uniform. It can be seen that the construction of sports grounds in primary and secondary schools in towns and villages in Nanchang is relatively poor, and it is an area that should be focused on in the future.

\section{References}

[1] Wang Hongzhi, Yin Yujia. Research on the Current Situation and Optimization Strategy of Public Sports Facilities in Guiyang City[J]. Journal of Guiyang University (Natural Science Edition), 2016, 11(03): 60-63+68.

[2] Zhou Zhaojun, Li Pan. Research on GIS-based layout of urban emergency shelters[J].Bulletin of Science and Technology,2016,32(05):202-206.

[3] Na Juhua. Research on Spatial Distribution Application of National Fitness Facilities Based on GIS_-Taking Weifang City of Shandong Province as an Example[J].Shandong Sports Science and Technology, 2015,37(05):116-118.

[4] WANG Xiaolin, CUI Mengyu, FU Peng. Planning and Design of Rural Sports Facilities Based on GIS Technology[J]. China Management Informationization, 2015,18(17):174-175. 